\title{
The primitive element theorem for differential fields with zero derivation on the ground field
}

\author{
Gleb A. Pogudin ${ }^{\mathrm{a}}$ \\ ${ }^{a}$ Department of Higher Algebra, Faculty of Mechanics and Mathematics, Moscow State University, Leninskie Gory 1, GSP-1, \\ Moscow, 119991, Russia
}

\begin{abstract}
In this paper we strengthen Kolchin's theorem (1] $)$ in the ordinary case. It states that if a differential field $E$ is finitely generated over a differential subfield $F \subset E, \operatorname{trdeg}_{F} E<\infty$, and $F$ contains a nonconstant, i.e. an element $f$ such that $f^{\prime} \neq 0$, then there exists $a \in E$ such that $E$ is generated by $a$ and $F$. We replace the last condition with the existence of a nonconstant element in $E$.

Keywords: differential field, primitive element, derivation

2000 MSC: $12 \mathrm{H} 05,13 \mathrm{~N} 15$
\end{abstract}

\section{Introduction}

All fields considered in this paper are of characteristic zero.

Let $R$ be a ring. A map $D: R \rightarrow R$ satisfying $D(a+b)=D(a)+D(b)$ and $D(a b)=a D(b)+D(a) b$ for all $a, b \in R$ is called derivation. We will denote $D(x)$ by $x^{\prime}$ and $D^{n}(x)$ by $x^{(n)}$.

A differential ring $R$ is a ring with a specified derivation. A differential ring which is a field will be called a differential field. Let $F \subset E$ be a differential field extension and $a \in E$. Let us denote by $F\langle a\rangle$ the differential subfield of $E$ generated by $F$ and $a$. If $F\langle a\rangle=E$, then element $a$ is said to be primitive.

An element $a \in R$ of the differential ring $R$ is said to be constant if $a^{\prime}=0$.

Kolchin proved ([1]) a differential analogue of the primitive element theorem:

Theorem. Let $E=F\left\langle a_{1}, \ldots, a_{n}\right\rangle$ and $\operatorname{trdeg}_{F} E<\infty$. Assume also that $F$ contains a nonconstant element. Then, there exists $b \in E$ such that $E=F\langle b\rangle$.

Corollary. Let $E=F\left\langle a_{1}, \ldots, a_{n}\right\rangle$ and $\operatorname{trdeg}_{F} E<\infty$. Assume also that $E$ contains a nonconstant element. Then, there exist $b, c \in E$ such that $E=F\langle b, c\rangle$.

Remark 1. In 1] Kolchin considered a more general case, i.e. fields equipped with a set of commuting derivations. We restrict ourselves to the ordinary case.

In 2] Babakhanian constructed primitive elements for several specific extensions $F \subset E$ with $F$ consisting of constant elements.

The goal of the present paper is to prove the primitive element theorem for the case $f^{\prime}=0$ for all $f \in F$.

\section{Main results}

Theorem 1. Let $E=k\langle a, b\rangle, \operatorname{trdeg}_{k} E<\infty$, and $b^{\prime} \neq 0$. Then, there exists $p(x) \in k[x]$ such that $\operatorname{trdeg}_{k} k\langle a+$ $p(b)\rangle=\operatorname{trdeg}_{k} k\langle a, b\rangle$.

Email address: pogudin.gleb@gmail.com (Gleb A. Pogudin) 
Theorem 2. Let $E=k\left\langle a_{1}, \ldots, a_{m}\right\rangle, \operatorname{trdeg}_{k} E<\infty$, and $E$ contains a nonconstant. Then, there exists a $\in E$ such that $E=k\langle a\rangle$.

Remark 2. Unlike Kolchin's proof it is not sufficient to consider elements of the form $a+\lambda b(\lambda \in k)$. For example, let $\mathbb{Q}(x, y)$ be a differential field with the derivation defined by $x^{\prime}=1$ and $y^{\prime}=0$. There is no primitive element of the form $y+\lambda x(\lambda \in \mathbb{Q})$, but $\mathbb{Q}(x, y)=\mathbb{Q}\left\langle x^{2}+y\right\rangle$.

Proof of Theorem 1. We will need the following well-known lemmas:

Lemma 1. If $\operatorname{trdeg}_{k} k\langle a\rangle=n$, then $k\langle a\rangle=k\left(a, a^{\prime}, \ldots, a^{(n)}\right)$.

Proof. Let $m$ be the minimal integer such that $a, \ldots, a^{(m)}$ are algebraically dependent over $k$. Let $R\left(a, \ldots, a^{(m)}\right)=0$ be the corresponding algebraic relation. Hence

$$
0=\left(R\left(a, \ldots, a^{(m)}\right)\right)^{\prime}=\sum_{i=0}^{m} a^{(i+1)} \frac{\partial}{\partial a^{(i)}} R
$$

, so $a^{(m+1)} \in k\left(a, \ldots, a^{(m)}\right)$.

Similarly we obtain that $a^{(N)} \in k\left(a, \ldots, a^{(m)}\right)$ for all $N$. Hence, $n=m$ and $k\langle a\rangle=k\left(a, \ldots, a^{(n)}\right)$.

Lemma 2 (3], p.35). Let $q\left(x, x^{\prime}, \ldots, x^{(n)}\right)$ be a nonzero differential polynomial over a differential field E. Let $f \in E$ be a noncostant element. Then, there exists $p(t) \in \mathbb{Q}[t]$ such that

$$
\left.q\left(x, x^{\prime}, \ldots, x^{(n)}\right)\right|_{x=p(f)} \neq 0
$$

Without loss of generality, we can assume that $E=k\langle a, b\rangle$. Let us introduce algebraically independent variables $\Lambda_{0}, \Lambda_{1}, \ldots$. We extend the derivation from $E$ to $E\left[\Lambda_{0}, \Lambda_{1}, \ldots\right]$ by $\left(\Lambda_{i}\right)^{\prime}=b^{\prime} \Lambda_{i+1}$. This construction can be explained by the following observation: let us fix a polynomial $p(x) \in \mathbb{Q}[x]$; the formulas $\varphi_{p}\left(\Lambda_{i}\right)=p^{(i)}(b)$ define a homomorphism of differential $k$-algebras $\varphi_{p}: E\left[\Lambda_{0}, \Lambda_{1}, \ldots\right] \rightarrow E$.

Let $c=a+\Lambda_{0}$ and $K=k\left(\Lambda_{0}, \Lambda_{1}, \ldots\right) \subset E\left(\Lambda_{0}, \Lambda_{1}, \ldots\right)$. Since $K\langle c\rangle \subset K\langle a, b\rangle, \operatorname{trdeg}_{K} K\langle c\rangle=n<\infty$. Let nonzero $R\left(x_{0}, \ldots, x_{n}\right) \in K\left[x_{0}, \ldots, x_{n}\right]$ satisfy $R\left(c, c^{\prime}, \ldots, c^{(n)}\right)=0$. Notice that it depends on $x_{n}$. Multiplying by the suitable element of $k\left[\Lambda_{0}, \Lambda_{1}, \ldots\right]$, we obtain a polynomial in both $c, c^{\prime}, \ldots, c^{(n)}$ and $\Lambda_{0}, \ldots, \Lambda_{N}$ over $k$. Let us denote it by $Q\left(c, \ldots, c^{(n)}, \Lambda_{0}, \ldots, \Lambda_{N}\right)$. Moreover, we assume that $Q$ satisfies the following conditions:

1. $\operatorname{deg}_{c^{(n)}} Q$ is a minimal possible;

2. under the above condition, $N$ is a minimal possible;

3. under the above conditions, $\operatorname{deg}_{\Lambda_{N}} Q$ is minimal possible.

Lemma 3. $N=n$.

Proof. Assume that $N>n$. Let us rewrite $Q$ as a polynomial in $\Lambda_{N}: Q=q_{m} \Lambda_{N}^{m}+\ldots+q_{0}$, where $q_{i}$ are polynomials over $k$ in $c, \ldots, c^{(n)}, \Lambda_{0}, \ldots, \Lambda_{N-1}$. Since $N>n, c, \ldots, c^{(n)} \in E\left(\Lambda_{0}, \ldots, \Lambda_{N-1}\right)$ and $\Lambda_{N}$ is transcendental over $k\left(c, \ldots, c^{(n)}, \Lambda_{0}, \ldots, \Lambda_{N-1}\right)$. Thus, $Q=0$ implies $q_{i}=0$ for all $i$. We obtained a contradiction with minimality of $N$.

Assume that $N<n$. Clearly, $c^{(n)}=\left(b^{\prime}\right)^{n} \Lambda_{n}+c_{0}$, where $c_{0} \in E\left(\Lambda_{0}, \ldots, \Lambda_{n-1}\right)$. Thus, $c^{(n)}$ is transcendental over $k\left(c, \ldots, c^{(n-1)}, \Lambda_{0}, \ldots, \Lambda_{N}\right) \subset E\left(\Lambda_{0}, \ldots, \Lambda_{n-1}\right)$. But $Q$ depends on $c^{(n)}$. This contradiction proves the lemma.

Lemma 4. $\frac{\partial}{\partial \Lambda_{n}} Q \neq 0$. 
Proof. It follows immediately from the minimality conditions for $Q$ and inequalities $\operatorname{deg}_{c^{(n)}} Q \geqslant \operatorname{deg}_{c^{(n)}} \frac{\partial}{\partial \Lambda_{n}} Q$ and $\operatorname{deg}_{\Lambda_{n}} Q>\operatorname{deg}_{\Lambda_{n}} \frac{\partial}{\partial \Lambda_{n}} Q>-\infty$.

We return to the proof of Theorem [1.

Let $p(x) \in \mathbb{Q}[x]$. Applying $\varphi_{p}$ to $Q\left(c, \ldots, c^{(n)}, \Lambda_{0}, \ldots, \Lambda_{n}\right)$, we obtain an algebraic dependence for $\varphi_{p}\left(\Lambda_{0}\right), \ldots, \varphi_{p}\left(\Lambda_{n}\right) \in$ $\mathbb{Q}[b]$ over $k\left(\varphi_{p}(c), \ldots, \varphi_{p}\left(c^{(n)}\right)\right)$. It yields to an algebraic dependence for $b$ over $k\left(\varphi_{p}(c), \ldots, \varphi_{p}\left(c^{(n)}\right)\right)$. The goal is to find an appropriate $p(x)$ to make this dependence nontrivial. Let us compute its derivation with respect to $b$ :

$$
\begin{aligned}
\frac{\partial}{\partial b} Q\left(\varphi_{p}(c), \ldots, \varphi_{p}\left(c^{(n)}\right), p(b), \ldots, p^{(n)}(b)\right)= & \\
& =\sum_{i=0}^{n} \varphi_{p}\left(\frac{\partial}{\partial \Lambda_{i}} Q\right) p^{(i+1)}(b)=\varphi_{p}\left(\sum_{i=0}^{n} \Lambda_{i+1} \frac{\partial}{\partial \Lambda_{i}} Q\right)
\end{aligned}
$$

By Lemma 4 the polynomial $T=\sum_{i=0}^{n} \Lambda_{i+1} \frac{\partial}{\partial \Lambda_{i}} Q$ is nonzero. Since $c=a+\Lambda_{0}$, we can rewrite $T$ as a nonzero polynomial in $\Lambda_{0}, \ldots, \Lambda_{n+1}$ over $k\langle a, b\rangle$. Let us denote the derivation on $E$ by $D$. Then, $\tilde{D}=\frac{1}{b^{\prime}} D$ is also a derivation on $E$. Obviously, $\tilde{D} \Lambda_{i}=\Lambda_{i+1}$.

Hence, we can apply Lemma 2 to the differential field $E$ with respect to $\tilde{D}$, nonconstant element $b \in E$, and the polynomial $T$ in variables $\Lambda_{0}, \ldots, \Lambda_{n}$. Therefore, we obtain such $p(x) \in \mathbb{Q}[x]$ that $\varphi_{p}(T) \neq 0$.

Since $\varphi_{p}(c)=a+p(b)$ and $b$ are both algebraic over $k\left\langle\varphi_{p}(c)\right\rangle$, $a$ is also algebraic over $k\left\langle\varphi_{p}(c)\right\rangle$. Hence, $\operatorname{trdeg}_{k} k\left\langle\varphi_{p}(c)\right\rangle=\operatorname{trdeg} k\langle a, b\rangle$.

The following corollary can be derived using exactly the same argument as above.

Corollary 1. Let $E=k\langle a, b\rangle, \operatorname{trdeg}_{k} E<\infty, b^{\prime} \neq 0$ and $c \in k\langle b\rangle$. Then, there exists $p(x) \in k[x]$ such that $\operatorname{trdeg} k\langle a+c \cdot p(b)\rangle=\operatorname{trdeg} k\langle a, b\rangle$.

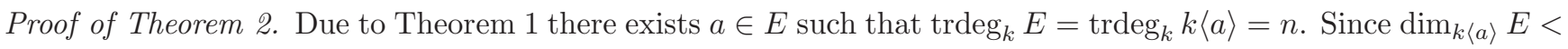
$\infty$ there exists $b \in E$ such that $E=k\langle a, b\rangle$. We are going to find $\lambda_{1}, \ldots, \lambda_{n+2} \in k$ such that $E=k\left\langle b+\lambda_{1} a+\right.$ $\left.\lambda_{2} a^{2}+\ldots+\lambda_{n+2} a^{n+2}\right\rangle$.

We will use the method used by Kolchin in [1, p.729]. Let us recall necessary definitions.

Let $K_{1}$ be a differential extension field of $L$. By an isomorphism of $K_{1}$ with respect to $L$ we will mean an isomorphic mapping of $K_{1}$ onto a differential field $K_{2}$ such that

1. $K_{2}$ is an extension of $L$;

2. the isomorphic mapping leaves each element of $L$ invariant;

Lemma 5 (Kolchin, (1] , p.726)). Let $E$ be an extension of $F$ and $\gamma \in E$. A necessary and sufficent condition for $E=F\langle\gamma\rangle$ is that no isomorphism of $E$ with respect to $F$ other then the identity leaves $\gamma$ invariant.

Let $I_{0}=\{f(x, y) \in k\{x, y\} \mid f(a, b)=0\}$ be the ideal of differential polynomials vanishing at $(a, b)$. We will show that there exist elements $\lambda_{1}, \ldots, \lambda_{n}$ such that $z=y+\lambda_{1} x+\ldots+\lambda_{n} x^{n}$ takes different values for different generic solutions of $I_{0}$ (see [1, p.725]). Then, certainly $z$ will satisfy the requirements on $\gamma$ from Lemma 5

To prove this statement, let $t_{1}, \ldots, t_{n+2}$ be new indeterminates, and, in $E\left\{x, y, t_{1}, \ldots, t_{n+2}\right\}$, consider the perfect differential ideal (for definitions see $[3, \mathrm{p} .2, \mathrm{p} .7]$ )

$$
I=\left\{I_{0}, t_{1}^{\prime}, \ldots, t_{n+2}^{\prime}, b-y+t_{1}(a-x)+t_{2}\left(a^{2}-x^{2}\right)+\ldots+t_{n+2}\left(a^{n+2}-x^{n+2}\right)\right\}
$$


Let $I=\mathfrak{p}_{1} \cap \ldots \cap \mathfrak{p}_{s}$ be the decomposition of $I$ into essential prime differential ideals (see [3, p.13]), and suppose the subscripts have been assigned so that for every $1 \leqslant i \leqslant r$ one of the following conditions hold

(C1) $a-x, b-y \in \mathfrak{p}_{i}$

(C2) $k\{x, y\} \cap \mathfrak{p}_{i} \neq I_{0}$.

Consider $\mathfrak{p}_{i}$ with $i \leqslant r$ and its solution $\left(\bar{x}, \bar{y}, \bar{t}_{1}, \ldots, \bar{t}_{n+2}\right)$. If $\left.(\mathrm{C} 1)\right]$ holds, then $(a, b)=(\bar{x}, \bar{y})$. If $(\mathrm{C} 2)$ holds, $(\bar{x}, \bar{y})$ is not a generic solution of $I_{0}$. Consider $\mathfrak{p}_{j}$ with $j>r$. If $b-y \notin \mathfrak{p}_{j}$, then also $a-x \notin \mathfrak{p}_{j}$. Thus, $a-x \notin \mathfrak{p}_{j}$ for all $j>r$. Let $\bar{x}, \bar{y}, \bar{t}_{1}, \ldots, \bar{t}_{n}$ be a generic solution of $\mathfrak{p}_{j}$. Then $(\bar{x}, \bar{y})$ is a generic solution of $I_{0}$. In particular, $\bar{y}$ is algebraic over $k\langle\bar{x}\rangle$. Differentiating the equation

$$
b-\bar{y}+\bar{t}_{1}(a-\bar{x})+\bar{t}_{2}\left(a^{2}-\bar{x}^{2}\right)+\ldots+\bar{t}_{n}\left(a^{n}-\bar{x}^{n}\right)=0
$$

$n+1$ times, we obtain a system of linear equations in $\bar{t}_{1}, \ldots, \bar{t}_{n+2}$. Let us investigate it.

Let us denote by $\operatorname{wr}\left(f_{1}, \ldots, f_{N}\right)$ the wronskian of $f_{1}, \ldots, f_{N}$ (see [4, chap. 2]).

Let $W_{k, l}(x, y)$ be given by $\operatorname{wr}\left(x-y, \ldots, \widehat{x^{l}-y^{l}}, \ldots, x^{k+1}-y^{k+1}\right)$ where $k \geqslant 2$ and $1 \leqslant l \leqslant k+1$.

Lemma 6. If $W_{k, l}(a, \bar{x})=0$ for all $1 \leqslant l \leqslant k+1$, then $\operatorname{trdeg}_{k} k\langle a, \bar{x}\rangle \leqslant n+k-2$.

Proof. Let $x$ and $y$ be differential indeterminates. First of all, we are going to establish several properties of differential polynomials $W_{k, l}(x, y)$.

Lemma 7. $W_{k, l}(x, y)=A_{l}(x, y)+x^{(k-1)} B_{l}(x, y)+y^{(k-1)} C_{l}(x, y)$ where $A_{l}, B_{l}, C_{l} \in \mathbb{Q}\left[x, \ldots, x^{(k-2)}, y, \ldots, y^{(k-2)}\right]$. Moreover, if $k \geqslant 3$, then $B_{l}(x, y)=-y^{\prime} D_{l}(x, y)$ and $C_{l}(x, y)=x^{\prime} D_{l}(x, y)$ where $D_{l} \in \mathbb{Q}\left[x, \ldots, x^{(k-2)}, y, \ldots, y^{(k-2)}\right]$.

Proof. For the sake of simplicity let us consider $l=k+1$. The proof for the other cases is analogous. The last row of the corresponding matrix is a sum of three rows: $x^{(k-1)}\left(1,2 x, \ldots, k x^{k-1}\right),-y^{(k-1)}\left(1,2 y, \ldots, y^{k-1}\right)$ and $\left(a_{1}, \ldots, a_{k}\right)$ where $a_{i} \in \mathbb{Q}\left[x, \ldots, x^{(k-2)}, y, \ldots, y^{(k-2)}\right]$ for all $i$. Thus, the determinant $W_{k, k+1}(x, y)$ can be expressed as a sum:

$$
\left|\begin{array}{ccc}
x-y & \ldots & x^{k}-y^{k} \\
\vdots & \ddots & \vdots \\
(x-y)^{(k-2)} & \ldots & \left(x^{k}-y^{k}\right)^{(k-2)} \\
a_{1} & \ldots & a_{k}
\end{array}\right|+x^{(k-1)}\left|\begin{array}{ccc}
x-y & \ldots & x^{k}-y^{k} \\
\vdots & \ddots & \vdots \\
(x-y)^{(k-2)} & \ldots & \left(x^{k}-y^{k}\right)^{(k-2)} \\
1 & \ldots & k x^{k-1}
\end{array}\right|-y^{(k-1)}\left|\begin{array}{ccc}
x-y & \ldots & x^{k}-y^{k} \\
\vdots & \ddots & \vdots \\
(x-y)^{(k-2)} & \ldots & \left(x^{k}-y^{k}\right)^{(k-2)} \\
1 & \ldots & k y^{k-1}
\end{array}\right|
$$

The above equality proves the first statement of the lemma.

Now let $k \geqslant 3$. The second row of the corresponding matrix is a sum of $x^{\prime}\left(1,2 x, \ldots, k x^{k-1}\right)$ and $-y^{\prime}\left(1,2 y, \ldots, k y^{k-1}\right)$. Hence, substracting the last row from the second, we obtain:

$$
B_{l}=\left|\begin{array}{ccc}
x-y & \ldots & x^{k}-y^{k} \\
x^{\prime}-y^{\prime} & \ldots & x^{\prime} k x^{k-1}-y^{\prime} k y^{k-1} \\
\vdots & \ddots & \vdots \\
1 & \ldots & k x^{k-1}
\end{array}\right|=-y^{\prime}\left|\begin{array}{ccc}
x-y & \ldots & x^{k}-y^{k} \\
1 & \ldots & k y^{k-1} \\
\vdots & \ddots & \vdots \\
1 & \ldots & k x^{k-1}
\end{array}\right|
$$

Let us denote the latter determinant by $D_{l}$. Then, $B_{l}=-y^{\prime} D_{l}$. Likewise, $C_{l}=x^{\prime} D_{l}$, so we are done.

Lemma 8. At least one of $\frac{W_{k, 1}(x, y)}{W_{k, k+1}(x, y)}, \ldots, \frac{W_{k, k}(x, y)}{W_{k, k+1}(x, y)}$ depends on $x^{(k-1)}$ and $y^{(k-1)}$.

Proof. Since all these differential polynomials are symmetric in $x$ and $y$, it suffices to prove that at least one of them depends on either $x^{(k-1)}$ or $y^{(k-1)}$. Assume the contrary, that $(-1)^{k-l} \frac{W_{k, l}(x, y)}{W_{k, k+1}(x, y)} \in \mathbb{Q}\left(x, \ldots, x^{(k-2)}, y, \ldots, y^{(k-2)}\right)$. By the Cramer's rule these fractions are solutions of the following system of linear equations in $\alpha_{1}, \ldots, \alpha_{k}$ : 


$$
\left(\begin{array}{ccc}
x-y & \cdots & x^{k}-y^{k} \\
\vdots & \ddots & \vdots \\
(x-y)^{(k-1)} & \ldots & \left(x^{k}-y^{k}\right)^{(k-1)}
\end{array}\right)\left(\begin{array}{c}
\alpha_{1} \\
\vdots \\
\alpha_{k}
\end{array}\right)=\left(\begin{array}{c}
x^{k+1}-y^{k+1} \\
\vdots \\
\left(x^{k+1}-y^{k+1}\right)^{(k-1)}
\end{array}\right)
$$

Since $\alpha_{1}, \ldots, \alpha_{k} \in \mathbb{Q}\left(x, \ldots, x^{(k-2)}, y, \ldots, y^{(k-2)}\right)$ and both $x^{(k-1)}$ and $y^{(k-1)}$ are transcendental over this field, the last equation also implies two following equalities:

$$
\left\{\begin{array}{l}
\alpha_{1}+2 x \alpha_{2}+\ldots+k x^{k-1} \alpha_{k}=(k+1) x^{k} \\
\alpha_{1}+2 y \alpha_{2}+\ldots+k y^{k-1} \alpha_{k}=(k+1) y^{k}
\end{array}\right.
$$

We are going to assign values from a particular differential field to $x$ and $y$. Precisely, let $\mathbb{C}(t)$ be a field of rational functions equipped with standard derivation $\left(t^{\prime}=1\right)$ and $\xi$ be a primitive $(k+1)$-th root of unity. Let $x=t$ and $y=\xi t$. The matrix of the system $\mathbb{*}$ is nondegenerate, because its determinant equals wr $\left((1-\xi) t, \ldots,\left(1-\xi^{k}\right) t^{k}\right)$, which is nonzero since $(1-\xi) t, \ldots,\left(1-\xi^{k}\right) t^{k}$ are linearly independent over constants (44, prop. 2.8]). Clearly, the unique solution of the system 因 in this case is $\alpha_{1}=\ldots=\alpha_{k}=0$. But the equalities (***) do not hold. This contradiction proves the lemma.

Corollary 2. If $k \geqslant 3$ then there exists $1 \leqslant l \leqslant k$ such that $W_{k, l}(x, y) D_{k+1}(x, y)-W_{k, k+1}(x, y) D_{l}(x, y)=$ $A_{l}(x, y) D_{k+1}(x, y)-A_{k+1}(x, y) D_{l}(x, y) \neq 0$.

Proof. By the Lemma 8 there exists $1 \leqslant l \leqslant k$ such that $\frac{W_{k, l}(x, y)}{W_{k, k+1}(x, y)}$ depends on $x^{(k-1)}$ and $y^{(k-1)}$. This means that vectors $\left(A_{l}, B_{l}, C_{l}\right)$ and $\left(A_{k+1}, B_{k+1}, C_{k+1}\right)$ are not proportional. Thus, $D_{k+1}\left(A_{l}, B_{l}, C_{l}\right)-D_{l}\left(A_{k+1}, B_{k+1}, C_{k+1}\right)=$ $\left(A_{l} D_{k+1}-A_{k+1} D_{l}, 0,0\right) \neq 0$.

We return to the proof of Lemma 6 ,

Let us consider two cases:

1. $k \leqslant 3$. Let $l$ be the index from the corollary 2. Since $W_{k, l}(a, \bar{x})=W_{k, k+1}(a, \bar{x})=0, W_{k, l}(a, \bar{x}) D_{k+1}(a, \bar{x})-$ $W_{k, k+1}(a, \bar{x}) D_{l}(a, \bar{x})$ provides us an algebraic relation between $a, \ldots, a^{(k-2)}, \bar{x}, \ldots, \bar{x}^{(k-2)}$. Hence, $\bar{x}^{(j)}$ is algebraic over $k\left(a, \ldots, a^{(n-1)}, \bar{x}, \ldots, \bar{x}^{(k-3)}\right)$ for all $j$. Thus, $k\langle a, \bar{x}\rangle$ is algebraic over $k\left(a, \ldots, a^{(n-1)}, \bar{x}, \ldots, \bar{x}^{(k-3)}\right)$. Since $\operatorname{trdeg}_{k} k\left(a, \ldots, a^{(n-1)}, \bar{x}, \ldots, \bar{x}^{(k-3)}\right) \leqslant n+k-2$, we are done.

2. $k=2$. In this case both $W_{2,2}(x, y)$ and $W_{2,3}(x, y)$ can be computed directly:

$$
\begin{gathered}
W_{2,3}(x, y)=(x-y)^{2}\left(x^{\prime}+y^{\prime}\right) \\
W_{2,2}(x, y)=(x-y)\left(x^{\prime}\left(2 x^{2}-x y-y^{2}\right)+y^{\prime}\left(x^{2}+x y-2 y^{2}\right)\right)
\end{gathered}
$$

If both $W_{2,3}(a, \bar{x})$ and $W_{2,2}(a, \bar{x})$ vanish, either $a^{\prime}=\bar{x}^{\prime}=0$ or the determinant of the system of linear equations $W_{2,3}(a, \bar{x})=W_{2,2}(a, \bar{x})=0$ in variables $a^{\prime}$ and $\bar{x}^{\prime}$ vanishes, i.e. $-(a-\bar{x})^{5}=0$. Both cases are impossible since $a \neq \bar{x}$.

Lemma 9. $\operatorname{trdeg}_{k} k\left\langle a, b, \bar{x}, \bar{y}, \bar{t}_{1}, \ldots, \bar{t}_{n+2}\right\rangle \leqslant 2 n+1$.

Proof. Differentiating the equation $\bar{y}-b=\bar{t}_{1}(a-\bar{x})+\ldots+\bar{t}_{n+2}\left(a^{n+2}-\bar{x}^{n+2}\right)$, we obtain the following matrix equality: 


$$
\left(\begin{array}{c}
\bar{y}-b \\
\bar{y}^{\prime}-b^{\prime} \\
\vdots \\
\bar{y}^{(n+1)}-b^{(n+1)}
\end{array}\right)=\left(\begin{array}{ccc}
a-\bar{x} & \ldots & a^{n+2}-\bar{x}^{n+2} \\
a^{\prime}-\bar{x}^{\prime} & \ldots & \left(a^{n+2}-\bar{x}^{n+2}\right)^{\prime} \\
\vdots & \ddots & \vdots \\
a^{(n+1)}-\bar{x}^{(n+1)} & \ldots & \left(a^{n+2}-\bar{x}^{n+2}\right)^{(n+1)}
\end{array}\right)\left(\begin{array}{c}
\bar{t}_{1} \\
\bar{t}_{2} \\
\vdots \\
\bar{t}_{n+2}
\end{array}\right)
$$

Let $k$ be a minimal number such that for all $1 \leqslant l \leqslant k+1$ the equality $W_{k, l}(a, \bar{x})=0$ holds. Let us consider two cases:

1. $k<n+2$. Thus, at least one of $(k-1) \times(k-1)$ minors of the matrix:

$$
\left(\begin{array}{ccc}
a-\bar{x} & \cdots & a^{n+2}-\bar{x}^{n+2} \\
\cdots & \ddots & \vdots \\
a^{(k-2)}-\bar{x}^{(k-2)} & \cdots & \left(a^{n+2}-\bar{x}^{n+2}\right)^{(k-2)}
\end{array}\right)
$$

is nondegenerate. Let $W_{k-1, l}(a, \bar{x}) \neq 0$. Multiplying by the inverse matrix, we obtain the formulas which express $\bar{t}_{j}$ as a rational function in $a, b, \bar{x}, \bar{y}$ and their derivations, $\bar{t}_{l}, \bar{t}_{k+1}, \ldots, \bar{t}_{n+2}$ for all $1 \leqslant j \leqslant k$ and $j \neq l$.

Hence, $\bar{t}_{1}, \ldots, \bar{t}_{l-1}, \bar{t}_{l+1}, \ldots, \bar{t}_{k} \in k\langle a, b, \bar{x}, \bar{y}\rangle\left(\bar{t}_{l}, \bar{t}_{k+1}, \ldots, \bar{t}_{n+2}\right)$. By Lemma 6 , $\operatorname{trdeg}_{k} k\langle a, b, \bar{x}, \bar{y}\rangle \leqslant n+k-2$. Thus

$$
\operatorname{trdeg}_{k} k\left\langle a, b, \bar{x}, \bar{y}, \bar{t}_{1}, \ldots, \bar{t}_{n+2}\right\rangle \leqslant \operatorname{trdeg}_{k} k\langle a, b, \bar{x}, \bar{y}\rangle+n-k+3 \leqslant(n+k-2)+(n-k+3)=2 n+1
$$

2. $k \geqslant n+2$. In this case, $\operatorname{trdeg}_{k} k\langle a, b, \bar{x}, \bar{y}\rangle \leqslant 2 n$. There exists $1 \leqslant l \leqslant n+2$ such that $W_{n+1, l}(a, \bar{x}) \neq 0$. By the same argument as above $\bar{t}_{1}, \ldots, \bar{t}_{l-1}, \bar{t}_{l+1}, \ldots, \bar{t}_{n+2} \in k\langle a, b, \bar{x}, \bar{y}\rangle\left(\bar{t}_{l}\right)$. The desired inequality is now obvious.

Lemma 9 implies that $\bar{t}_{1}, \ldots, \bar{t}_{n+2}$ are algebraically dependent over $k\langle a, b\rangle$. Let us denote this dependence by $P_{j}\left(t_{1}, \ldots, t_{n+2}\right) \in E\left[t_{1}, \ldots t_{n+2}\right]$. Consider the polynomial $P=P_{r+1} \cdot \ldots \cdot P_{s}$. Let $\lambda_{1}, \ldots, \lambda_{n+2} \in k$ satisfy $P\left(\lambda_{1}, \ldots, \lambda_{n+2}\right) \neq 0$. Then, $b-y+\lambda_{1}(a-x)+\ldots+\lambda_{n+2}\left(a^{n+2}-a^{n+2}\right) \neq 0$ for any generic solution of $I_{0}$ other than $(b, a)$. Therefore, the proof of the theorem is complete.

The author is grateful to Dmitry Trushin, Yu.P. Razmyslow, and Lei Fu for useful discussions.

[1] Kolchin E.R., Extensions of differential fields, I, Annals of Mathematics, vol. 43, 1942.

[2] Babakhanian A., On primitive elements in differentially algebraic extension fields, Trans. AMS, vol. 143, 71-83, 1968.

[3] Ritt J.F., Differential algebra, Colloquium publications of AMS, vol. 33, 1948.

[4] Magid A. R., Lectures on differential Galois theory, University lecture series of AMS, vol. 7, 1994. 